\title{
Selectivity in Postencoding Connectivity with High-Level Visual Cortex Is Associated with Reward-Motivated Memory
}

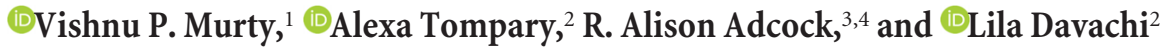 \\ ${ }^{1}$ Department of Psychiatry, University of Pittsburgh, Pittsburgh, Pennsylvania 15213, ${ }^{2}$ Department of Psychology, New York University, New York, \\ New York 10003, ${ }^{3}$ Center for Cognitive Neuroscience, Duke University, Durham, North Carolina 27708, and ${ }^{4}$ Department of Psychiatry and Behavioral \\ Science, Duke University, Durham, North Carolina 27708
}

Reward motivation has been demonstrated to enhance declarative memory by facilitating systems-level consolidation. Although highreward information is often intermixed with lower reward information during an experience, memory for high value information is prioritized. How is this selectivity achieved? One possibility is that postencoding consolidation processes bias memory strengthening to those representations associated with higher reward. To test this hypothesis, we investigated the influence of differential reward motivation on the selectivity of postencoding markers of systems-level memory consolidation. Human participants encoded intermixed, trial-unique memoranda that were associated with either high or low-value during fMRI acquisition. Encoding was interleaved with periods of rest, allowing us to investigate experience-dependent changes in connectivity as they related to later memory. Behaviorally, we found that reward motivation enhanced $24 \mathrm{~h}$ associative memory. Analysis of patterns of postencoding connectivity showed that, even though learning trials were intermixed, there was significantly greater connectivity with regions of high-level, category-selective visual cortex associated with high-reward trials. Specifically, increased connectivity of category-selective visual cortex with both the VTA and the anterior hippocampus predicted associative memory for high- but not low-reward memories. Critically, these results were independent of encoding-related connectivity and univariate activity measures. Thus, these findings support a model by which the selective stabilization of memories for salient events is supported by postencoding interactions with sensory cortex associated with reward.

Key words: category-selective visual cortex; consolidation; hippocampus; rest; reward; VTA

Significance Statement

Reward motivation is thought to promote memory by supporting memory consolidation. Yet, little is known as to how brain selects relevant information for subsequent consolidation based on reward. We show that experience-dependent changes in connectivity of both the anterior hippocampus and the VTA with high-level visual cortex selectively predicts memory for highreward memoranda at a $24 \mathrm{~h}$ delay. These findings provide evidence for a novel mechanism guiding the consolidation of memories for valuable events, namely, postencoding interactions between neural systems supporting mesolimbic dopamine activation, episodic memory, and perception.

\section{Introduction}

It is adaptive for individuals to store valuable information in long-term memory. Although information is often intermixed in experience, memory systems have the capacity to prioritize mem-

Received Nov. 9, 2015; revised Aug. 2, 2016; accepted Sept. 7, 2016.

Author contributions: V.P.M., R.A.A., and L.D. designed research; V.P.M. performed research; V.P.M., A.T., and L.D. analyzed data; V.P.M., A.T., R.A.A., and L.D. wrote the paper.

This work was supported by DART Neuroscience to L.D., National Institutes of Health Grant R01 MH074692 to L.D., and National Institutes of Health Grant F32 DA036361 to V.P.M. We thank Issa Barakat for assistance in data collection and analysis.

The authors declare no competing financial interests.

Correspondence should be addressed to Dr. Lila Davachi, New York University, Department of Psychology, 6 Washington Place, Room 871, New York, NY 10003. E-mail: lila.davachi@nyu.edu.

DOI:10.1523/JNEUROSCI.4032-15.2016

Copyright $\odot 2017$ the authors $\quad 0270-6474 / 17 / 370537-09 \$ 15.00 / 0$ ory for high-value information. Recent work has begun to characterize how reward promotes episodic memory. Extant research has emphasized the role of the hippocampus and the mesolimbic dopamine system, such as the VTA, before (Adcock et al., 2006) and during encoding of rewarded memoranda (Wittmann et al., 2005; Wolosin et al., 2012). Behavioral work in rodents and humans has shown that, when memory for rewarded information is tested immediately after encoding, there is often no facilitation or only a modest improvement; rather, the benefits emerge after a significant delay (Wang and Morris, 2010; Murayama and Kuhbandner, 2011; Murayama and Kitagami, 2014). This delay-dependent facilitation suggests that selectivity of high-reward memories must be, at least in part, supported by postencoding consolidation. However, less is known regarding the neural mechanisms underlying the pri- 
oritization of valuable information in long-term memory by consolidation.

One mechanism that may support individuals' ability to selectively prioritize highvalue information is systems-level memory consolidation. Within this framework, episodic associations are initially encoded within the hippocampus and become stabilized into long-term memory through postencoding interactions between the hippocampus and sensory cortex associated with initial encoding (McClelland et al., 1995; Nadel et al., 2000; Sutherland and McNaughton, 2000; Davachi and Danker, 2013; Davachi and Preston, 2014). Recent studies have provided empirical support for this notion by demonstrating that postencoding connectivity of the hippocampus with high-level, category-selective visual cortex (CSC) predicts later associative memory (Tambini et al., 2010; Schlichting and Preston, 2014; Tompary et al., 2015). In regards to high-value information, mechanisms of systems-level memory consolidation may be centered on the anterior hippocampus, as prior research has implicated the anterior hippocampus in memory for affective/motivationally relevant information (Poppenk et al., 2013). If postencoding connectivity reflects coordinated hippocampal-cortical processing, this could provide a substrate for the prioritization of high-value information in long-term memory.

In addition to hippocampally mediated consolidation, previous research in animal models has implicated the mesolimbic system in postencoding mechanisms of memory consolidation (Wang and Morris, 2010). In rodent, introducing rewarding and novel events after encoding strengthens hippocampus-dependent memory when memory is tested at a significant delay (Moncada and Viola, 2007; Wang et al., 2010; Salvetti et al., 2014). Furthermore, manipulation of mesolimbic function via drug administration has been demonstrated to influence delayed, but not immediate, tests of hippocampal-dependent memory (O'Carroll et al., 2006; Rossato et al., 2009; Bethus et al., 2010). These memory enhancements have mainly been attributed to increases in cellular consolidation mechanisms, such as stabilized hippocampal synaptic plasticity (Lisman et al., 2011). In line with these animal models, recent research in humans has shown that postencoding interactions between the VTA and hippocampus predict reward-motivated memory enhancements (Gruber et al., 2016). Although these prior studies demonstrate memory enhancements for events co-occurring with or preceding mesolimbic engagement, they do not necessarily provide mechanistic insight into how systems-level consolidation may selectively stabilize into long-term memory. One possibility is that postencoding interactions of the VTA with sensory cortex could support the selective memory stabilization of valuable representations. Although interactions between mesolimbic systems and sensory cortex have been documented (Berger et al., 1990; Lidow et al., 1991; Arsenault et al., 2013; Zaldivar et al., 2014), research has yet to investi- gate whether these interactions support consolidation in the service of prioritizing valuable information.

Given previous behavioral evidence for the selective consolidation for high-value items, we tested whether markers of systems-level consolidation, involving postencoding interactions with sensory cortex, uniquely contribute to memory for highversus low-value information. During the acquisition of fMRI data, participants encoded intermixed, trial-unique pictureword pairs (Fig. 1). Critically, encoding was interleaved with periods of rest, allowing us to investigate experience-dependent changes in connectivity as they related to memory on a $24 \mathrm{~h}$ delayed surprise memory test. Critically, high- and low-reward memoranda were always drawn from distinct visual categories within a given subject (i.e., either all faces or scenes), allowing us to directly test the hypothesis that reward would selectively enhance systems-level consolidation in sensory cortex previously associated with higher reward.

\section{Materials and Methods}

\section{Participants}

Participants were recruited from the New York University and New York City communities for an fMRI study. Informed consent was obtained from each participant in a manner approved by the University Committee on Activities Involving Human Subjects. Twenty healthy, right-handed participants (12 females; age range: $18-34$ years; median age $=23$ years) were each paid $\$ 60$ to participate. 


\section{Experimental procedures}

Before scanning, participants were given instructions for the encoding, retrieval, and localizer task. Additionally, participants performed a short practice of the encoding and immediate retrieval task. Inside the scanner, participants first performed the baseline resting-state scan followed by the localizer task. Participants then completed 3 rounds of the following sequence of scans: encoding, retrieval, and resting state. After a $24 \mathrm{~h}$ delay, participants returned to a behavioral testing room and performed a surprise associative retrieval memory task.

Resting-state scan. Participants completed a resting-state scan before and following each run of reward-motivated encoding. During the resting-state scan, participants were instructed to keep their eyes closed during the scan while trying to remain awake. In a postscan query, all participants reported staying awake during rest-scans.

Localizer task. Following the baseline rest scan, participants completed a localizer task to obtain independent ROIs in the fusiform face area (FFA) and parahippocampal place area (PPA). During the localizer task, participants completed a target detection task. They were instructed to press a button whenever they detected an inverted image. During this task, participants viewed blocks of trial-unique places or faces. During each block participants viewed 16 pictures (either all faces or places) for $0.4 \mathrm{~s}$ each with an interstimulus interval of $0.4 \mathrm{~s}$. Within each block, either 1 or 2 images were vertically inverted. Following each block, participants viewed a fixation cross for $16 \mathrm{~s}$. Participants received 7 blocks of face and place objects, respectively, in a pseudo-randomized order such that there was no more than 2 subsequent repetitions of one condition.

Reward-motivated memory task. Following the baseline rest scan, participants completed three runs of a rewarded-motivated encoding task (Fig. 1). Each trial began with the presentation of a cue for $1 \mathrm{~s}$ indicating the condition (i.e., high or low-reward), followed by a fixation dot for 2-6 s, and then an encoding phase for $4 \mathrm{~s}$. During the encoding phase, participants were presented with a trial-unique image and adjective and were instructed to visualize a scenario in which the adjective described the image. Following the encoding phase, participants viewed a fixation cross on average for $8.4 \mathrm{~s}$ (range: 3-21 s) until the next trial started. Participants were instructed that their memory for a subset of the associations would be tested immediately after each encoding run (retrieval task is described below). Further, they were informed that successful memory for associations following a high-reward cue worked toward earning a $\$ 20$ bonus, whereas successful memory for associations following a low-reward cue worked toward earning a $\$ 1$ bonus. Participants received 22 high-reward trials and 22 low-reward trials within each of the three encoding runs.

To test how high-level visual, category-specific cortex contributes to reward-motivated encoding, trial-unique images were drawn from distinct categories for high- and low-reward conditions. For half of the participants, the high-reward condition consisted of celebrity faces and the low-reward condition consisted of famous places. In the other half of the participants, categories were switched across reward conditions. Face and place stimuli did not differ in terms of subjective ratings of how recognizable the images were in a separate behavioral cohort $(p=0.91)$.

Following each encoding run, participants completed a short associative recognition task on a subset of stimuli selected from the previous run of encoding ( 2 high-reward trials, 2 low-reward trials). Participants were given explicit instructions that only object-adjective pairs that immediately preceded the retrieval test would appear in the test. This instruction ensured that there was no explicit incentive to rehearse object-adjective pairs during postencoding rest. Thus, these immediate memory tests reflected an experimental manipulation, as opposed to an assay of memory for items. During each trial, participants were shown a single image and three adjectives from the previous encoding phase. Participants were instructed to indicate which adjective was previously paired with the presented image. Participants had $20 \mathrm{~s}$ to indicate a response. We used a liberal response threshold of $20 \mathrm{~s}$ because we did not want participants to focus on the speed of their response at the expense of memory accuracy. Each trial was followed by a fixation cross for $0.5 \mathrm{~s}$. Trial order was randomized. Critically, participants were instructed that performance on this associative recognition test would determine their monetary bonus such that correctly answering $66 \%$ of high- and low-reward trials would result in a $\$ 20$ and $\$ 1$ bonus, respectively.

Participants returned $24 \mathrm{~h}$ later for a surprise retrieval test in a purely behavioral session. During this session, we tested participants' associative cued-recall, associative recognition, and specificity for object images. Before initiating the task, participants were instructed that this session was not incentivized. During each trial, participants were shown two object images on the top of the screen: an object image from the encoding phase and a novel exemplar of the same image (i.e., another picture of the celebrity or a picture of the famous place from another view). Participants were asked three questions about each image pair. First, during the associative cued-recall phase, participants were presented with three word stems (i.e., the first two letters of previously encoded adjectives) and were instructed to recall which adjective was associated with the object image. They indicated their response by selecting which of three wordstems matched the word they recalled: if the participant recalled the adjective 'bright' they would select 'br.' Participants could also indicate a 'don't know' response. Memory accuracy was calculated as a measure of associative hit rate ([associative hits/(associative hits + associative misses)]) separately for pairs in the high- and low-reward condition. We chose to use this measure of associative hit rate, as opposed to percentage correct associative hits, to characterize memory, because this measure accounts for (1) any differences that may have emerged from changes in item memory across conditions (i.e., a don't know response could indicate unknown item memory or associative memory) and (2) potential category-level differences in the rate at which individuals are likely to endorse an associative answer across categories of stimuli/reward conditions (e.g., participants were more likely to use a don't know response for faces vs scenes). Participants additionally performed an associative recognition test and item specificity test. We only analyzed the associative cued-recall memory data, as prior research has suggested this measure may be the most sensitive to hippocampal function (Habib and Nyberg, 2008). Each trial was followed by a fixation cross for $0.5 \mathrm{~s}$. All object-adjectives pairs that were not tested immediately were presented during this phase in a randomized order (60 high-reward associations, 60 low-reward associations)

\section{MRI data acquisition and preprocessing}

Functional imaging data were acquired on a Siemens Allegra 3T headonly scanner with a custom head coil (NM-011; Nova Medical) using an EPI pulse sequence $(\mathrm{TE}=15 \mathrm{~ms} ; 82$ degrees; $\mathrm{TR}=2000 \mathrm{~ms} ; 34$ interleaved slices; voxel size $=3 \times 3 \times 3 \mathrm{~mm}$ ). Slices were positioned parallel to the AC-PC axis and included whole-brain coverage, except for the most superior portions of motor and parietal cortices. Resting-state scans were 244 volumes, the localizer scan was 212 volumes, and encoding scans were 422 volumes. Following collection of the functional scans, we collected a high-resolution T1-weighted anatomical scan (magnetization-prepared rapid-acquisition gradient echo sequence; voxel size $=$ isotropic $1 \mathrm{~mm}$ ) for use in spatial normalization.

Before fMRI preprocessing, data were inspected on custom software for head motion and scanning artifacts. Data were analyzed only if they exhibited $<3.0 \mathrm{~mm}$ motion (absolute maximum). Slice acquisitions with isolated transient noise artifacts (i.e., scanner spiking) were replaced with interpolated data from neighboring time points. fMRI preprocessing was then performed using fMRI Expert Analysis Tool, version 6.00 as implemented in FSL 5.0.2.1. The first 4 scans of each run were discarded to allow for signal saturation. BOLD images were then skull stripped using the Brain Extraction Tool. Images were then realigned withinrun, intensity normalized by a single multiplicative factor, spatially smoothed with a $5.0 \mathrm{~mm}$ FWHM kernel, and subjected to a high-pass filter (Gaussian-weighted least-squares straight line fitting, with $\sigma=$ $100 \mathrm{~s})$. Preprocessed functional imaging data remained in subjectspecific space; however, normalization and coregistration parameters were estimated to transform localizer ROIs into the space of resting-state scans.

For the resting-state scans, which are especially sensitive to noise from head motion, we implemented additional preprocessing procedures. First, for each voxel's time series, we regressed out the variance tied to 6 parameters describing individual subject's volume-to-volume motion 
(3 rotation parameters, 3 translation parameters). Second, we identified and removed outlier volumes in our functional data. We identified outlier volumes by evaluating the root-mean-square error (RMSE) of each volume relative to the reference volume (the middle time point). We considered a volume an outlier if its RMSE amplitude exceeded the 75 percentile plus the value of the $150 \%$ of the interquartile range of RMSE for all volumes in a run (i.e., a standard boxplot threshold). We removed these outlier time points with frame-specific regressors in a GLM (i.e., on regressor per outlier).

\section{ROI definition}

We wanted to investigate resting-state connectivity among four regions implicated in our reward-motivated encoding paradigm: VTA, hippocampus, FFA, and PPA. The VTA was defined in standard space using a probabilistic atlas thresholded to $75 \%$ (Murty et al., 2014). The hippocampus was defined in standard space using a probabilistic atlas thresholded at 75\% defined from the Harvard-Oxford probablistic subcortical atlas as implemented by fsl (https://fsl.fmrib.ox.ac.uk/fsl/fslwiki/Atlases). To generate anterior and posterior hippocampal ROIs, we divided the original hippocampal ROI on the long-axis and used the most anterior and posterior tertile. For each individual participant, these ROIs were transformed into subject-specific space using the inverse of the parameters estimated during normalization.

The FFA and PPA were localized for individual subjects using contrasts from the localizer scans (PPA: Places $>$ Faces; FFA: Faces $>$ Places). Specifically, ROIs were defined as $4 \mathrm{~mm}$ spheres centered around peak coordinates within the fusiform face area and parahippocampal place area, respectively. Peak coordinates were identified in subject-specific space by a trained research assistant, and verified by the first author.

The FFA and PPA were then assigned as either high-reward or lowreward CSC based on the reward conditions of each participant, which was counterbalanced across participants (see Fig. 3). For example, for the 10 even participants for which the high-reward memoranda were celebrity faces, FFA was assigned as the high-reward CSC and PPA as the low-reward CSC. For the 10 odd participants with high-reward condition of famous places, PPA was assigned as high-reward CSC and FFA as low-reward CSC.

\section{Resting-state analysis}

To investigate changes in functional connectivity across baseline and postencoding resting-state scans, we performed seed-based functional connectivity analyses. For each rest scan, we extracted the mean timeseries from the rest scans for each ROI (PPA, FFA, hippocampus, VTA; defined above). These time-series were then submitted to pairwise Pearson correlations separately for each rest scan. To get a single measure of postencoding resting-state connectivity, we averaged correlation scores (i.e., $r$-scores) across all three postencoding rest scans. To look for differences in connectivity as a function of reward-motivated encoding, we submitted postencoding correlations and baseline correlations to a paired $t$ test.

Further, to investigate how changes in resting-state connectivity was related to reward-motivated memory, we ran Pearson partial correlations as implemented by MATLAB 2014a partialcorr function (MathWorks) between connectivity changes (postencoding - baseline) and associative cued-recall, separately for high- and low-reward conditions, while controlling for group (i.e., High-reward = Scene or Highreward $=$ Face). Data were considered significant at a threshold of $p<0.05$, two-tailed.

To compare differences in correlations across the high- and lowreward conditions, we submitted correlation coefficients to an $r$-to- $z$ transform while accounting for the repeated measures and dependence across the two correlations. For this analysis, data were considered significant at a threshold of $p<0.05$, one-tailed, given the post hoc/directional nature of these tests.

Although the $r$-to- $z$ transform analysis is appropriate to compare two correlations within a single ROI pair, it is not well suited to compare reward-related differences in correlations across difference sets of ROI pairs (i.e., Is the difference between high- and low-reward brainbehavior correlations larger for pair A-X vs B-X?). To perform this latter statistical test, we conducted a permutation-based analysis following Duncan et al. (2014). Specifically, a nonparametric shuffle approach was used to test for an interaction between region-pair and reward condition (high, low). To develop a null distribution, we randomly shuffled the region pair label within participants $(\mathrm{A}-\mathrm{X}, \mathrm{B}-\mathrm{X})$ and calculated the brainbehavior relationships between each pair on this shuffled data. We then calculated the interaction term (A-X High-reward - A-X Lowreward) - (B-X High-reward - B-X Low-reward). We repeated this procedure 100,000 times to develop a null distribution for the interaction term. Finally, we calculated the interaction term in our unshuffled intact data and calculated the proportion of shuffled data that resulted in an interaction term with an absolute value as large as was observed in our intact dataset.

\section{Control analysis for univariate activation during encoding}

We ran control analyses to determine whether postencoding relationships of functional coupling and memory performance were independent of univariate activation in response to reward cues and during encoding. Encoding fMRI data were preprocessed using the same parameters described for resting-state scans. Next, we estimated univariate activation in the VTA and the hippocampus during encoding in response to reward cues and during encoding (i.e., in response to memoranda). Specifically, we constructed first-level (within-run) GLMs that included 4 regressors that modeled high-reward cues, low-reward cues, high-reward encoding, and low-reward encoding. Cue regressors were modeled with event durations of $1 \mathrm{~s}$, and encoding regressors were modeled with event durations of $4 \mathrm{~s}$. All regressors were convolved with a double- $\gamma$ hemodynamic response function. Using this GLM, individual maps of parameter estimates were generated for 4 contrasts of interest: high-reward cue $>$ intrinsic baseline, low-reward cue $>$ intrinsic baseline, high-reward encoding $>$ intrinsic baseline, low-reward encoding $>$ intrinsic baseline. The intrinsic baseline reflects nonmodeled portions of the task, which only included fixation intervals. Second-level analyses for each of these contrasts (i.e., across runs, within-subject) were modeled using a fixedeffects analysis. Parameter estimates from these contrasts were extracted from the VTA, the hippocampus, the anterior hippocampus, and the posterior hippocampus ROIs and averaged across all three encoding runs for each individual subject. These values were used in partial regressions between resting-state coupling and memory performance as implemented by MATLAB 2014a partialcorr function.

\section{Control analysis for connectivity during encoding}

We next ran control analyses to determine whether postencoding relationships of functional coupling and memory performance were independent of functional coupling during encoding. Functional coupling was measured using a "background connectivity" approach during encoding, which is the most similar to functional coupling measures used during rest (Al-Aidroos et al., 2012). This approach has previously been used to look at how functional coupling measures across participants is related to $24 \mathrm{~h}$ consolidated memories (Duncan et al., 2014). In short, we first removed trial-evoked activity and nuisance signals using the voxelwise GLM approach described above. We then extracted time-series from the filtered residuals and calculated pairwise coupling between ROIs. We then averaged these correlation scores across all three encoding runs for each individual subject. These values were used in partial regressions between resting-state coupling and memory performance as implemented by MATLAB 2014a partialcorr function.

\section{Results}

\section{Behavioral performance}

After encoding, participants returned $24 \mathrm{~h}$ later to complete a nonincentivized, surprise cued-recall memory test for pictureword pairs encoded in high- versus low-reward conditions. They were shown a picture and instructed to recall the associated word. Reward motivation resulted in a better associative memory, as measured by associative hit rate ([associative hits/(associative hits + associative misses)]), for pairs encoded in the high-reward condition (high $>$ low associative hit rate; $t_{(19)}=2.58 ; p=0.02$; 


\section{Associative Recall Test}

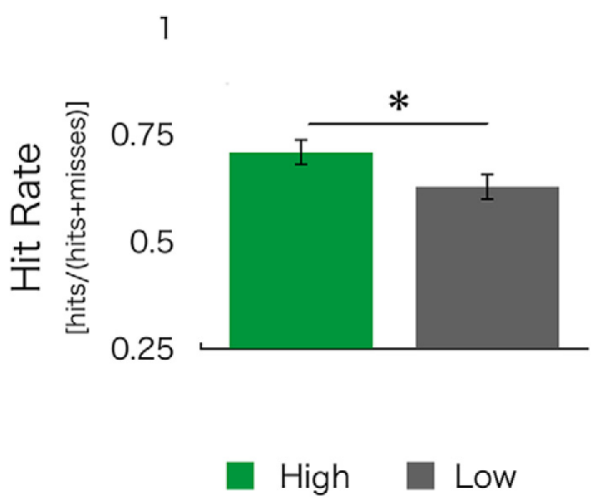

Figure 2. Motivated encoding influences $24 \mathrm{~h}$ associative memory. At a $24 \mathrm{~h}$ test, associative cued recall was greater for pairs encoded under high- versus low-reward motivation. Critically, participants showed a selectivity of memory for high-value information despite retrieval being nonincentivized. ${ }^{*} p<0.05$.

Fig. 2). Specifically, participants' associative hit rate was higher in the high- versus low-reward condition (high: $46.1 \%$; low: $35.3 \%$; high $>$ low: $t_{(19)}=3.00, p=0.01$ ), without any significant differences in misses (high: 19.9\%; low: 22.2\%; high $>$ low: $t_{(19)}=$ $-1.20, p=0.25)$.

\section{Experience-dependent changes in functional connectivity}

To investigate whether postencoding consolidation may support the selective stabilization of valuable information, we measured experience-dependent changes in functional connectivity of sensory cortex with the hippocampus and the VTA. Because we were particularly interested in whether reward dynamically targets the processing of items associated with reward in high-level visual cortex, we selected targets in high-level, CSC associated with processing faces and scenes (i.e., the FFA and the PPA, respectively), where high-reward was associated with faces/FFA in half of our participants and with scenes/PPA in the other half (Fig. 3).

For each pair of regions, we first investigated whether connectivity changed with experience as a function of reward-motivated encoding by comparing connectivity between postencoding and baseline rest scans. We next compared whether experiencedependent changes in resting-state connectivity (postencoding $>$ pre-encoding baseline) were related to individual differences in long-term associative cued recall, separately for high- and lowvalue word-picture pairs. To increase power, we collapsed across groups and categorized ROIs as high-reward-CSC (FFA in even subjects, PPA in odd subjects) and low-reward-CSC (PPA in even subjects, FFA in odd subjects). Of note, we controlled for group as a covariate of no interest in all analyses (see Materials and Methods).

\section{Hippocampus and CSC}

We first investigated whether reward-motivated encoding was associated with an increase in postencoding functional connectivity between the hippocampus and CSC. Although hippocampal connectivity did not, at the group level, increase with high- or low-reward CSC after encoding (high reward: $r \pm \mathrm{SEM}=$ $-0.05 \pm 0.02, p=0.09$; low reward: $r \pm \mathrm{SEM}=-0.04 \pm 0.02$, $p=0.11$, difference: $p=0.88$ ), we did see a relationship between experience-dependent changes in connectivity (postencoding $>$ pre-encoding baseline) and individual memory performance. Specifically, when using the entire hippocampal ROI, we found a significant positive correlation between hippocampal connectivity with high-reward CSC and associative memory for highreward pairs $\left(r_{(18)}=0.58, p=0.009\right)$. Likewise, a similar near significant positive correlation was seen between hippocampal connectivity with low-reward CSC and low-reward associative memory $\left(r_{(18)}=0.42, p=0.07\right)$. However, neither of these findings was specific to targets in CSC, as hippocampal-high-reward CSC interactions also showed a trend-level association with lowreward memory $\left(r_{(18)}=0.40, p=0.08\right)$ and hippocampal-lowreward CSC interactions showed a trend level association with high-reward memory $\left(r_{(18)}=0.43, p=0.06\right)$. Thus, although interactions between hippocampus, as a whole, and CSC predicted associative memory, these relationships were nonspecific and are not sufficient to explain mechanisms of rewardmotivated memory consolidation.

However, prior research has suggested functional specialization along the anterior-posterior hippocampal axis with anterior portions of the hippocampus hypothesized to preferentially contribute to reward-motivated memory (Poppenk et al., 2013). Thus, we next investigated a-priori, anatomically defined regions of the anterior versus posterior hippocampus. These two regions showed differential relationships between connectivity with CSC and memory for high- versus low-reward associations. We found a significant double dissociation (permutation-based testing: $p=0.01$ ) such that experience-dependent connectivity of the anterior hippocampus and CSC positively correlated with high, but not low, reward memory (high memory with high CSC: $r_{(18)}=0.51, p=0.02$; low memory with low CSC: $r_{(18)}=0.16$, $p=0.50$, difference in correlation: $p<0.05$, one-tailed; Figure 3 ), whereas experience-dependent connectivity of the posterior hippocampus with CSC positively correlated with low, but not high, reward memory (high memory with high CSC: $r_{(18)}=0.35, p=$ 0.14 ; low memory with low CSC: $r_{(18)}=0.63, p=0.004$, difference in correlation: $p<0.05$, one-tailed; Figure 3$)$. These findings suggest that sensory regions in the visual cortex interact with discrete regions of the hippocampus to support high- and lowvalue information.

\section{VTA and CSC}

Although systems-level consolidation classically refers to hippocampal-cortical connectivity, we additionally looked at VTA-cortical connectivity given the role of mesolimbic activation in reward consolidation. We found experience-dependent increases in resting-state connectivity (postencoding $>$ preencoding baseline) with the VTA in both high- and low-reward CSC (high-reward: $t_{(19)}=2.35, p=0.03$; low-reward: $t_{(19)}=$ 2.92, $p=0.009$ ), with no significant differences between the two (difference: $t_{(19)}=-1.17, p=0.28$ ). However, across-subjects analyses revealed that experience-dependent changes in connectivity of the VTA with high-reward CSC selectively correlated with associative cued recall for high-reward pairs $\left(r_{(18)}=0.46\right.$, $p=0.05$; Fig. 4), whereas no such relationship was evident between VTA-low-reward CSC connectivity and low-reward associative cued recall $\left(r_{(18)}=0.04, p=0.87\right.$; difference in correlation: $p<0.05$, one-tailed; Fig. 4$)$. This finding suggests that, after encoding, the VTA interacts with sensory cortex associated with reward to support the stabilization of longterm memories.

\section{Specificity of reward effects to postencoding connectivity}

Previous research has demonstrated that univariate VTA and hippocampal BOLD activation as well as connectivity before (Adcock et al., 2006) and during encoding (Wolosin et al., 2012) 
Category-Selective Cortex (CSC)

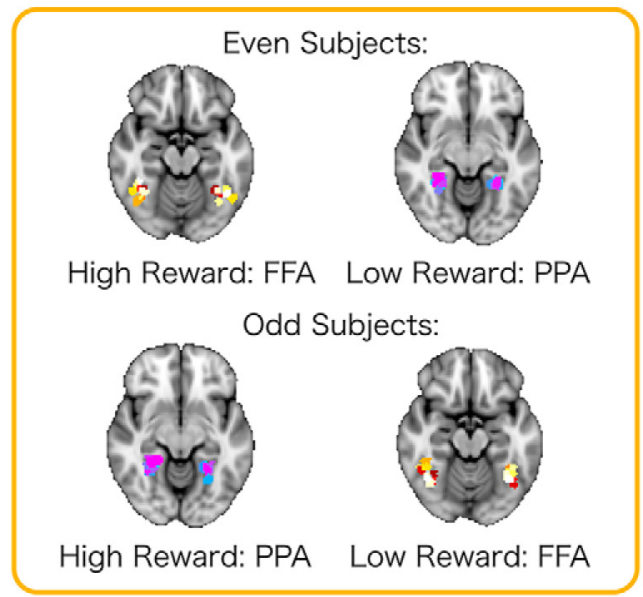

Hippocampus

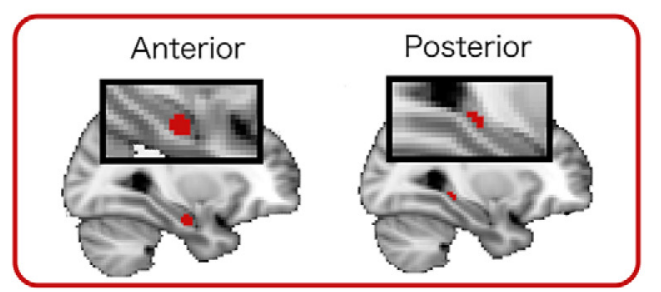

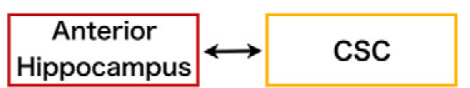
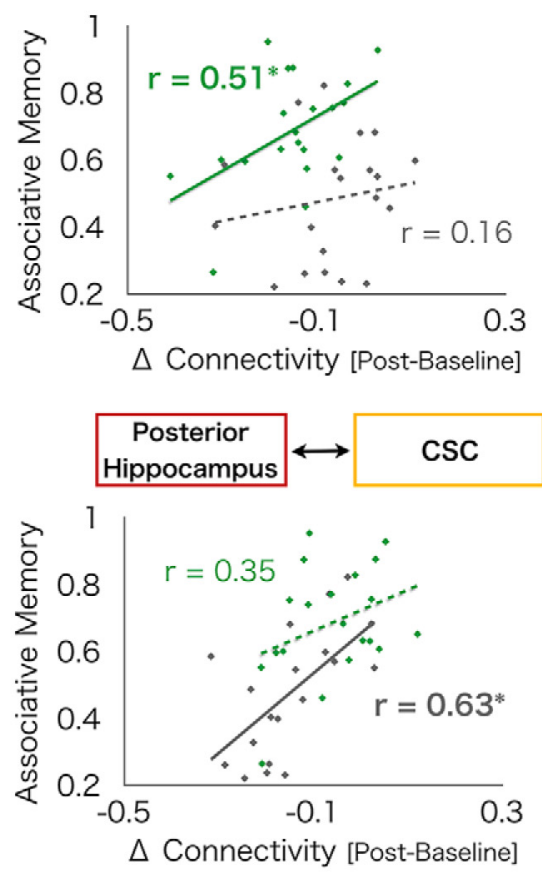

High Reward $\square$ Low Reward

Figure 3. Postencoding interactions between the hippocampus and CSC predict $24 \mathrm{~h}$ associative memory. Experience-dependent changes in coupling (postencoding $>$ baseline) of CSC (top left) with anterior and posterior hippocampus (bottom left) differentially predict high- and low-reward memory. Interactions between anterior hippocampus with high-reward CSC predict high-reward memory, although no such relationship exists between anterior hippocampus-low-reward CSC interactions and low-reward memory (top right). Conversely, interactions between posterior hippocampus with low-reward CSC predict low-reward memory, although no such relationship exists between posterior hippocampus-high-reward CSC interactions and high-reward memory (bottom right). High-and low-reward CSC refers to CSC associated with high-and low-rewards during encoding, respectively. Green dots indicate high-reward values. Gray dots indicates low-reward values. Solid lines indicate significant correlations. ${ }^{*} p<0.05$. Hyphenated lines indicate nonsignificant correlations.

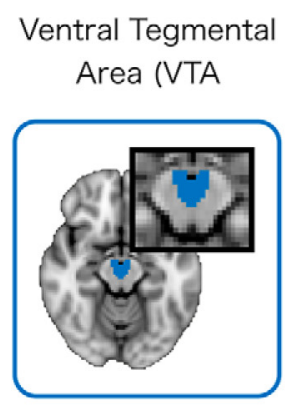

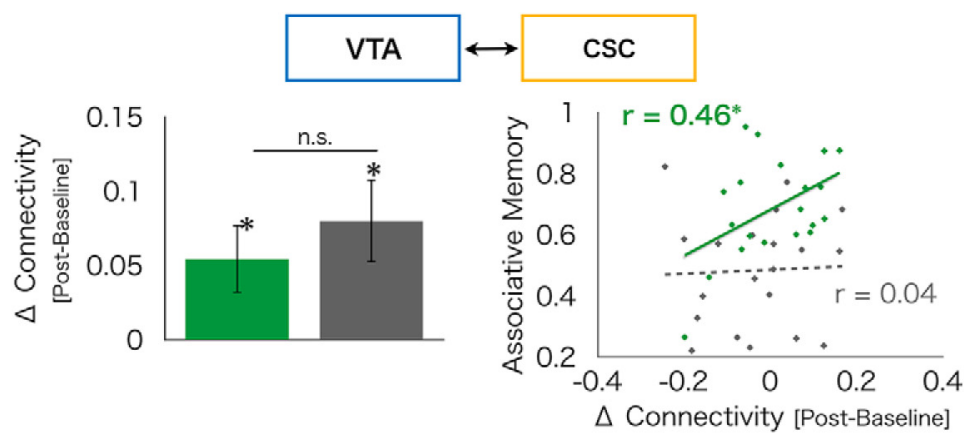

High Reward Low Reward

Figure 4. Postencoding interactions between the VTA and CSC. We found experience-dependent increases in functional connectivity of the VTA (left) with both high-reward CSC and low-reward CSC (middle). However, interactions of VTA-high-reward CSC predicted high-reward associative memory, although no such relationship exists between VTA-low-reward CSC interactions and low-reward memories (right). Green dots indicate high-reward values. Gray dots indicates low-reward values. Solid lines indicate significant correlations. ${ }^{*} p<0.05$. Hyphenated lines indicate nonsignificant correlations.

are related to memory for high, but not low, reward-motivated memoranda. Thus, we wanted to determine whether the postencoding results reported above are independent from both encoding-related activation and connectivity.

We found that relationships between CSC connectivity with the anterior hippocampus and high-reward memory remained significant when accounting for cue- and encoding-related activation, and remained numerically in the same direction when correcting for encoding-related connectivity but were nonsignificant ( $p=0.11$ ). Further, all relationships between CSC connectivity with the poste- rior hippocampus and low-reward memory remained significant when accounting for these measures. Further, the association between VTA-high-reward CSC postencoding connectivity and high-reward memory performance remained significant when accounting for univariate activation in the VTA both in response to reward cues and during stimulus encoding as well as VTA-CSC connectivity during encoding (Table 1). Together, these control analyses show that the relationships between postencoding connectivity and reward memory performance are independent of activity and connectivity during encoding. 
Table 1. Control analysis for encoding-related activation and connectivity

\begin{tabular}{|c|c|c|c|c|c|}
\hline Postencoding coupling & Covariate for partial regression & High reward $(r)$ & High reward $(p)$ & Low reward $(r)$ & Low reward $(p)$ \\
\hline \multirow[t]{3}{*}{ Anterior hippocampus-CSC } & Anterior hippocampus activation (cue) & 0.53 & $0.02^{*}$ & 0.18 & 0.48 \\
\hline & Anterior hippocampus activation (encoding) & 0.49 & $0.04^{*}$ & 0.13 & 0.61 \\
\hline & Anterior hippocampus-CSC encoding coupling & 0.39 & 0.11 & 0.17 & 0.5 \\
\hline \multirow{2}{*}{ Posterior hippocampus-CSC } & Posterior hippocampus activation (encoding) & 0.37 & 0.13 & 0.71 & $0.001^{*}$ \\
\hline & Posterior hippocampus-CSC encoding coupling & 0.32 & 0.2 & 0.6 & $0.008^{*}$ \\
\hline VTA-CSC & VTA activation (cue) & 0.5 & $0.03^{*}$ & 0.12 & 0.64 \\
\hline
\end{tabular}

${ }^{*} p<0.05$.

\section{Discussion}

Individuals have the capacity to prioritize high-value information into long-term memory. However, this selectivity of high-value information is strengthened over temporal delays (Murayama and Kuhbandner, 2011; Murayama and Kitagami, 2014), indicating a critical role for memory consolidation in stabilizing high-value memories. Little is known, however, as to how postencoding processes support this selectivity in reward memory. In the current study, we show that experience-dependent changes in resting-state connectivity selectively predict memory for high- versus low-value information. Behaviorally, we found that individuals had better memory for information associated with high reward during encoding. These memory enhancements emerged even though delayed memory tests were not explicitly incentivized, suggesting that reward may facilitate long-term memory for information that may be relevant to future adaptive behavior (Shohamy and Adcock, 2010; Lisman et al., 2011). Analysis of postencoding brain activity revealed changes in functional coupling of the anterior hippocampus and the VTA with CSC that predict memory for high-value, but not lowvalue, information. For example, when high-value information contained faces, we found that shifts in face-selective visual cortex (i.e., the FFA) predicted long-term memory. These results provide evidence that one mechanism by which our memory systems selectively stabilize memories for valuable information is by targeting sensory cortex associated with reward during postencoding consolidation.

Classic models of systems-level consolidation have proposed that interactions of the hippocampus with sensory cortex support memory consolidation (McClelland et al., 1995; Nadel et al., 2000; Sutherland and McNaughton, 2000) but have not previously been investigated as a mechanism of reward-based memory enhancements. Recent work using human neuroimaging has provided a putative marker of this type of consolidation processes: experience-dependent changes in functional coupling of the hippocampus (Tambini et al., 2010; van Kesteren et al., 2010; Schlichting and Preston, 2014; Tompary et al., 2015). For example, previous work from our laboratory has demonstrated that increases in hippocampal-cortical connectivity after encoding predicted associative memory for nonrewarded information (Tambini et al., 2010). Research has yet to investigate, however, how systems-level consolidation may support the selection of high- versus low-reward information.

When investigating the hippocampus in its entirety, we found that coupling with CSC was related to enhanced memory for both high- and low-value events. Further, additional analyses revealed that these relationships were nonspecific to the content of memoranda, and that characterizing the hippocampus as a unitary structure was insufficient in explaining mechanisms of enhanced consolidation for rewarded information. However, we found that subregions of the hippocampus differentially contrib- uted to memory for high- and low-value information during postencoding rest. We found a significant double-dissociation such that postencoding coupling of the anterior hippocampus with CSC uniquely predicted memory for high-value information, whereas coupling of the posterior hippocampus uniquely predicted memory for low-value information. Because we did not include a no-value condition, our current experimental design could not discriminate between the posterior hippocampus' role in the consolidation specifically for low-value information or for neutral information more generally. However, given prior animal and human literatures (reviewed below), we predict that the posterior hippocampus would similarly support consolidation of both low-value information and neutral information. These findings offer a plausible mechanism by which selectivity of reward emerges from the facilitation of postencoding consolidation mechanisms in hippocampal subcomponents. Consolidation processes facilitating neutral information may be limited to posterior portions of the hippocampus, whereas the selectivity of reward may result from targeting processes in the anterior portions of the hippocampus.

Interestingly, associations of anterior hippocampus with high-reward memory is consistent with results from rodent research that suggest that the ventral hippocampus, the rodent homolog to the human anterior hippocampus, is specialized to support memory for motivationally relevant information, such as affect, novelty, and reward (Fanselow and Dong, 2010). Similarly, in humans, the anterior hippocampus has been reliably associated with the successful encoding of motivationally relevant memoranda compared with neutral memoranda (Dolcos et al., 2004; Adcock et al., 2006; Murty et al., 2010; Poppenk et al., 2013). Importantly, these prior findings were limited to studies investigating memory encoding and/or retrieval. Thus, our current findings extend prior literatures by demonstrating a selective role for anterior hippocampus in supporting memories for motivationally relevant information to include postencoding consolidation.

Beyond the hippocampus, we found that experience-dependent changes between VTA and CSC predicted memory for high-value information. Further, these findings were selective to the high-reward condition as there was no such relationship for low-reward memory. Prior work has supported a role for the VTA in supporting selective consolidation for high-reward information. In humans, postencoding antagonism of dopamine, a neurotransmitter tightly coupled with VTA activation, has been shown to disrupt the selective consolidation of reward memories (Feld et al., 2014). Additionally, recent research in rodents has shown increased "replay" of patterns of prior reward-related activity in the VTA during rest (Singer and Frank, 2009; Valdés et al., 2015), and it has been proposed that these "replay" events 
support systems-level consolidation. Although the VTA has received much attention for its role in supporting systems-level consolidation of reward memory, research to date has not focused on how the VTA coordinates with cortical regions to achieve this. Thus, our findings provide support for a novel mechanism by which memories for valuable information are facilitated: interactions of the VTA with CSC. Emerging models of consolidation suggest that consolidation may only occur within cortex that receives a "salience tag" temporally proximal to encoding (Moncada et al., 2015), raising the intriguing, yet untested, hypothesis that functional interactions between the VTA and sensory cortex may only influence memory if a behavioral tag exists within the cortex.

Interestingly, the majority of studies investigating reward memory in humans have mainly focused on encoding, rather than postencoding processes (but see Oudiette et al., 2013; Gruber et al., 2016). These studies have demonstrated that memory for rewarding events is supported by activation and functional connectivity of the hippocampus and the VTA during encoding (Wittmann et al., 2005; Adcock et al., 2006; Callan and Schweighofer, 2008; Wolosin et al., 2012). Critically, we ran a series of control analyses demonstrating that our postencoding connectivity findings were independent of these encodingrelated markers of high-reward memory. All of our postencoding results replicated in models that controlled for individual variation in activation and connectivity of the VTA and the hippocampus, except that interactions between anterior hippocampus and CSC may be sensitive to encoding-related connectivity.

Thus, our findings predominately support a novel mechanism whereby postencoding processes contribute to the selection of high-reward representations for memory stabilization that is independent from previously demonstrated encoding-related activations (Wittmann et al., 2005; Adcock et al., 2006; Wolosin et al., 2012). Critically, the processes that support consolidation may be initiated during reward-motivated encoding, such as interactions between the VTA, the prefrontal cortex, and the hippocampus (Adcock et al., 2006; Murty and Adcock, 2014). Notably, we included a short memory probe of only a few trials before the postencoding rest period to eliminate rehearsal as a potential strategy adopted by our participants. Even though only a few trials, our current design will not allow us to discriminate whether our shifts in resting-state connectivity were modulated (either enhanced or dampened) by the presence of these few immediate retrieval trials. Given the prominent role for VTA activation and associated mesolimbic engagement in facilitating encoding/consolidation rather than retrieval (Wang and Morris, 2010), we think it is unlikely that our results are driven primarily by the retrieval trials. However, at a more fine-grained level, retrieval operations are always occurring during novel encoding and new encoding is occurring during retrieval so the broader issue of whether encoding or retrieval differentially modulates postencoding connectivity measures is an interesting topic that can be addressed with future work.

Although prior work has demonstrated that individuals have the capacity to prioritize high-value information into memory via consolidation, relatively less research has investigated the mechanisms supporting this selection. We find evidence for the selective stabilization of valuable information in memory by strengthening connectivity with sensory regions that represent information associated with reward. We provide evidence for two complimentary mechanisms of stabilizing high-value information after encoding: (1) a classically defined mechanisms of systems-level consolidation (i.e., hippocampal-cortical interac- tions); and (2) a novel mechanism of VTA neuromodulation over sensory cortex. Together, our results broaden models of both systems-level consolidation and reward memory.

\section{References}

Adcock RA, Thangavel A, Whitfield-Gabrieli S, Knutson B, Gabrieli JD (2006) Reward-motivated learning: mesolimbic activation precedes memory formation. Neuron 50:507-517. CrossRef Medline

Al-Aidroos N, Said CP, Turk-Browne NB (2012) Top-down attention switches coupling between low-level and high-level areas of human visual cortex. Proc Natl Acad Sci U S A 109:14675-14680. CrossRef Medline

Arsenault JT, Nelissen K, Jarraya B, Vanduffel W (2013) Dopaminergic reward signals selectively decrease fMRI activity in primate visual cortex. Neuron 77:1174-1186. CrossRef Medline

Berger B, Febvret A, Greengard P, Goldman-Rakic PS (1990) DARPP-32, a phosphoprotein enriched in dopaminoceptive neurons bearing dopamine D1 receptors: distribution in the cerebral cortex of the newborn and adult rhesus monkey. J Comp Neurol 299:327-348. CrossRef Medline

Bethus I, Tse D, Morris RG (2010) Dopamine and memory: modulation of the persistence of memory for novel hippocampal NMDA receptordependent paired associates. J Neurosci 30:1610-1618. CrossRef Medline

Callan DE, Schweighofer N (2008) Positive and negative modulation of word learning by reward anticipation. Hum Brain Mapp 29:237-249. CrossRef Medline

Davachi L, Danker JF (2013) Cognitive neuroscience of episodic memory. In: Oxford handbook of cognitive neuroscience, pp 375-388. Oxford: Oxford University.

Davachi L, Preston AR (2014) The medial temporal lobe and memory. In: The cognitive neurosciences, pp 539-546. Cambridge, MA: Massachusetts Institute of Technology.

Dolcos F, LaBar KS, Cabeza R (2004) Interaction between the amygdala and the medial temporal lobe memory system predicts better memory for emotional events. Neuron 42:855-863. CrossRef Medline

Duncan K, Tompary A, Davachi L (2014) Associative encoding and retrieval are predicted by functional connectivity in distinct hippocampal area CA1 pathways. J Neurosci 34:11188-11198. CrossRef Medline

Fanselow MS, Dong HW (2010) Are the dorsal and ventral hippocampus functionally distinct structures? Neuron 65:7-19. CrossRef Medline

Feld GB, Besedovsky L, Kaida K, Münte TF, Born J (2014) Dopamine D2like receptor activation wipes out preferential consolidation of high over low-reward memories during human sleep. J Cogn Neurosci 26:23102320. CrossRef Medline

Gruber MJ, Ritchey M, Wang SF, Doss MK, Ranganath C (2016) Postlearning hippocampal dynamics promote preferential retention of rewarding events. Neuron 89:1110-1120. CrossRef Medline

Habib R, Nyberg L (2008) Neural correlates of availability and accessibility in memory. Cereb Cortex 18:1720-1726. CrossRef Medline

Lidow MS, Goldman-Rakic PS, Gallager DW, Rakic P (1991) Distribution of dopaminergic receptors in the primate cerebral cortex: quantitative autoradiographic analysis using $\left[{ }^{3} \mathrm{H}\right]$ raclopride, $\left[{ }^{3} \mathrm{H}\right]$ spiperone and $\left[{ }^{3} \mathrm{H}\right] \mathrm{SCH} 23390$. Neuroscience 40:657-671. CrossRef Medline

Lisman J, Grace AA, Duzel E (2011) A neoHebbian framework for episodic memory: role of dopamine-dependent late LTP. Trends Neurosci 34: 536-547. CrossRef Medline

McClelland JL, McNaughton BL, O’Reilly RC (1995) Why there are complementary learning systems in the hippocampus and neocortex: insights from the successes and failures of connectionist models of learning and memory. Psychol Rev 102:419-457. CrossRef Medline

Moncada D, Viola H (2007) Induction of long-term memory by exposure to novelty requires protein synthesis: evidence for a behavioral tagging. J Neurosci 27:7476-7481. CrossRef Medline

Moncada D, Ballarini F, Viola H (2015) Behavioral tagging: a translation of the synaptic tagging and capture hypothesis. Neural Plast 2015:650780. CrossRef Medline

Murayama K, Kitagami S (2014) Consolidation power of extrinsic rewards: reward cues enhance long-term memory for irrelevant past events. J Exp Psychol Gen 143:15-20. CrossRef Medline

Murayama K, Kuhbandner C (2011) Money enhances memory consolidation-but only for boring material. Cognition 119:120-124. CrossRef Medline

Murty VP, Adcock RA (2014) Enriched encoding: reward motivation organizes cortical networks for hippocampal detection of unexpected events. Cereb Cortex 24:2160-2168. CrossRef Medline 
Murty VP, Ritchey M, Adcock RA, LaBar KS (2010) fMRI studies of successful emotional memory encoding: a quantitative meta-analysis. Neuropsychologia 48:3459-3469. CrossRef Medline

Murty VP, Shermohammed M, Smith DV, Carter RM, Huettel SA, Adcock RA (2014) Resting state networks distinguish human ventral tegmental area from substantia nigra. Neuroimage 100:580-589. CrossRef Medline

Nadel L, Samsonovich A, Ryan L, Moscovitch M (2000) Multiple trace theory of human memory: computational, neuroimaging, and neuropsychological results. Hippocampus 10:352-368. CrossRef Medline

O'Carroll CM, Martin SJ, Sandin J, Frenguelli B, Morris RG (2006) Dopaminergic modulation of the persistence of one-trial hippocampusdependent memory. Learn Mem 13:760-769. CrossRef Medline

Oudiette D, Antony JW, Creery JD, Paller KA (2013) The role of memory reactivation during wakefulness and sleep in determining which memories endure. J Neurosci 33:6672-6678. CrossRef Medline

Poppenk J, Evensmoen HR, Moscovitch M, Nadel L (2013) Long-axis specialization of the human hippocampus. Trends Cogn Sci 17:230-240. CrossRef Medline

Rossato JI, Bevilaqua LR, Izquierdo I, Medina JH, Cammarota M (2009) Dopamine controls persistence of long-term memory storage. Science 325:1017-1020. CrossRef Medline

Salvetti B, Morris RG, Wang SH (2014) The role of rewarding and novel events in facilitating memory persistence in a separate spatial memory task. Learn Mem 21:61-72. CrossRef Medline

Schlichting ML, Preston AR (2014) Memory reactivation during rest supports upcoming learning of related content. Proc Natl Acad Sci U S A 111:15845-15850. CrossRef Medline

Shohamy D, Adcock RA (2010) Dopamine and adaptive memory. Trends Cogn Sci 14:464-472. CrossRef Medline

Singer AC, Frank LM (2009) Rewarded outcomes enhance reactivation of experience in the hippocampus. Neuron 64:910-921. CrossRef Medline
Sutherland GR, McNaughton B (2000) Memory trace reactivation in hippocampal and neocortical neuronal ensembles. Curr Opin Neurobiol 10: 180-186. CrossRef Medline

Tambini A, Ketz N, Davachi L (2010) Enhanced brain correlations during rest are related to memory for recent experiences. Neuron 65:280-290. CrossRef Medline

Tompary A, Duncan K, Davachi L (2015) Consolidation of associative and item memory is related to post-encoding functional connectivity between the ventral tegmental area and different medial temporal lobe subregions during an unrelated task. J Neurosci 35:7326-7331. CrossRef Medline

Valdés JL, McNaughton BL, Fellous JM (2015) Offline reactivation of experience-dependent neuronal firing patterns in the rat ventral tegmental area. J Neurophysiol 114:1183-1195. CrossRef Medline

van Kesteren MT, Fernández G, Norris DG, Hermans EJ (2010) Persistent schema-dependent hippocampal-neocortical connectivity during memory encoding and postencoding rest in humans. Proc Natl Acad Sci U S A 107:7550-7555. CrossRef Medline

Wang SH, Morris RG (2010) Hippocampal-neocortical interactions in memory formation, consolidation, and reconsolidation. Annu Rev Psychol 61:49- 79, C1-C4. CrossRef Medline

Wittmann BC, Schott BH, Guderian S, Frey JU, Heinze HJ, Düzel E (2005) Reward-related FMRI activation of dopaminergic midbrain is associated with enhanced hippocampus-dependent long-term memory formation. Neuron 45:459-467. CrossRef Medline

Wolosin SM, Zeithamova D, Preston AR (2012) Reward modulation of hippocampal subfield activation during successful associative encoding and retrieval. J Cogn Neurosci 24:1532-1547. CrossRef Medline

Zaldivar D, Rauch A, Whittingstall K, Logothetis NK, Goense J (2014) Dopamine-induced dissociation of BOLD and neural activity in macaque visual cortex. Curr Biol 24:2805-2811. CrossRef Medline 